\title{
SMILE ASYMPTOTICS II: MODELS WITH KNOWN MOMENT GENERATING FUNCTIONS
}

\author{
SHALOM BENAIM * AND \\ PETER FRIZ, ${ }^{* * *}$ University of Cambridge
}

\begin{abstract}
The tail of risk neutral returns can be related explicitly with the wing behaviour of the Black-Scholes implied volatility smile. In situations where precise tail asymptotics are unknown but a moment generating function is available we establish, under easy-tocheck Tauberian conditions, tail asymptotics on logarithmic scales. Such asymptotics are enough to make the tail-wing formula (see Benaim and Friz (2008)) work and so we obtain, under generic conditions, a limiting slope when plotting the square of the implied volatility against the log strike, improving a lim sup statement obtained earlier by Lee (2004). We apply these results to time-changed exponential Lévy models and examine several popular models in more detail, both analytically and numerically.
\end{abstract}

Keywords: Implied volatility smile; tail-wing formula; Tauberian theory

2000 Mathematics Subject Classification: Primary 60E99; 91B70

\section{Introduction}

The purpose of this paper is to connect the tail behaviour of a real-valued random variable (viewed as returns in a stochastic financial model) to the wing behaviour of the Black-Scholes implied volatility, sharpening Lee's celebrated moment formula [8]. Let us recall that the famous Black-Scholes formula gives the unique price (based on no-arbitrage arguments) of a European call option (i.e. the option to buy a risky asset at some later time ('maturity') at a price ('strike') agreed today), based on the assumption that returns (or log price) of the asset follow a Brownian motion times a constant factor ('Black-Scholes volatility') plus a constant drift. For pricing purposes, we have to work under risk-neutral measure which, in particular, relates the drift to the risk-free rate. It is everyday practice in the financial industry to quote call prices seen in the market in terms of the 'implied' volatility, that is, the volatility parameter in the Black-Scholes formula that gives rise to a matching price. Of course, this implied volatility can and will depend on the maturity and strike of the call option under consideration. The implied volatility as a function of these two parameters is the famous volatility surface, e.g. [6] and [7], its dependence in strike often exhibits a smile ('volatility smile'). It is also everyday practice in the industry to fit the volatility surface to parametric models (typically diffusion or jump-diffusion processes). Not surprisingly then, the behaviour of the Black-Scholes implied volatility/variance at extreme strikes is of considerable interest to the financial industry: it constrains the functional form for the extrapolation of an implied volatility skew into the tails, and allows the inference of parameters of underlying dynamics, given observations of tail slopes of the volatility skew.

Received 15 February 2007; revision received 14 January 2008.

* Postal address: Statistical Laboratory, Centre for Mathematical Sciences, University of Cambridge, Wilberforce

Road, Cambridge CB3 0WB, UK.

** Email address: p.k.fritz@statslab.cam.ac.uk 
In a previous paper [4] motivated by Lee's moment formula [8], we established an explicit formula ('tail-wing formula') that relates the asymptotic behaviour of the tail of the distribution function of risk-neutral returns (for some fixed maturity) to the small-strike and large-strike asymptotics of the implied Black-Scholes volatility (the 'wings' of the implied volatility smile).

The present paper extends the discussion of [4] to models whose returns at some fixed maturity have a known moment generating function (MGF) $M$, but whose density $f$ (if it exists) and distribution function $F$ are, even asymptotically, unknown. For a large class of distributions used for modelling (risk neutral) returns in finance, $M$ is finite only on part of the real line. Let us define $\bar{F} \equiv 1-F$ and $r^{*}$ as the least upper bound of all real $r$ for which $M(r) \equiv \mathrm{E}\left[\mathrm{e}^{r X}\right]<\infty$, and assume that $r^{*} \in(0, \infty)$. An application of Chebyshev's inequality yields

$$
\lim \sup _{x \rightarrow \infty} \frac{-\log \bar{F}(x)}{x}=r^{*},
$$

and we may be tempted to conclude that

$$
-\log \bar{F}(x) \sim r^{*} x \quad \text { as } x \rightarrow \infty,
$$

where we use the standard notation $g(x) \sim h(x) \equiv \lim g(x) / h(x)=1$ as $x \rightarrow \infty$, but a simple counterexample shows that this is not true (it suffices to consider $\bar{F}(x):=\exp \left(-\mathrm{e}^{\log x}\right.$ ) as $x \rightarrow \infty)$. However, we do expect (2) to be true if the (right) tail of the distribution is somewhat reasonably behaved. Our interest in such distributions stems from the fact that the crude tail asymptotics (2) and the mild integrability condition $p^{*}=r^{*}-1>0$ are enough, via the tail-wing formula [4], to assert the existence of a limiting slope of Black-Scholes implied variance $V^{2}$ as a function of the $\log$ strike $k$ as $k$ tends to $\pm \infty$. Indeed, in standard notation, reviewed in Section 4, we have

$$
\lim _{k \rightarrow \infty} \frac{V^{2}(k)}{k}=2-4\left(\sqrt{\left(p^{*}\right)^{2}+p^{*}}-p^{*}\right) .
$$

Similarly, if $q^{*} \equiv \sup \left\{q \in \mathbb{R}: M(-q) \equiv \mathrm{E}\left[\mathrm{e}^{-q X}\right]<\infty\right\} \in(0, \infty)$ and the (left) tail is reasonably behaved, we expect $\log F(-x) \sim-q^{*} x$ as $x \rightarrow \infty$, in which case the tail-wing formula gives

$$
\lim _{k \rightarrow \infty} \frac{V^{2}(-k)}{k}=2-4\left(\sqrt{\left(q^{*}\right)^{2}+q^{*}}-q^{*}\right) .
$$

Quite similar to the discussion between (1) and (2), there is an elementary derivation [8] of (3) and (4) with lim sup instead of lim, while the corresponding limit statements are false in general. Nonetheless, we may hope that every reasonable asset model creates marginal distributions which do not exhibit oscillations in the far-out-of-the-money regions of the volatility surface. In fact, we should be reluctant to call a model reasonable if it has such oscillations. The main part of this paper is devoted to developing criteria that will allow us to check that a given model is 'reasonable'. Our working assumption, satisfied by a large class of financial models, is some knowledge of the MGF of the marginal laws. It will be seen in Section 3 that (2), and then (3) and (4) as a consequence, holds if the MGF behaves 'reasonably' at its endpoint of definition $r^{*}$ (and similarly for $-q^{*}$ ). The right condition on 'reasonable behaviour', which covers all our examples, will be given in terms of regular variation. That said, we cannot help expressing our surprise that we were unable to find a simple statement of the form

$$
r^{*} \equiv \sup \{s: M(s)<\infty\} \in(0, \infty)+\text { condition } \quad \Longrightarrow \quad-\log \bar{F}(x) \sim r^{*} x,
$$


in the vast literature on Tauberian theory, at least not with a condition sufficient for our applications. (A statement of form (5) appears in a recent preprint [9] in the context of queueing theory. The author's conditions are significantly stronger than ours (see Criteria 1 and 2 in Section 3) and would rule out important examples such as the normal inverse Gaussian (NIG) model.) We remark that under the assumption of our Criterion 1, below, and the additional assumption that

$$
\bar{F}(x)=\int_{x}^{\infty} \mathrm{e}^{-r y} \mathrm{~d} U(y)
$$

for some $U \in R_{\rho}, \rho>0$, which admits an ultimately monotone density (that is, $\mathrm{d} U / \mathrm{d} y$ is monotone on $[x, \infty)$ for large enough $x$ ), we can obtain full asymptotics of $\bar{F}$ (see [3]). These will of course imply the crude asymptotics on $\log \bar{F}(x)$, which is all that we need to establish (3) and (4). If the condition on ultimate monotonicity of $U^{\prime}$ is omitted altogether, we can construct counterexamples to see that the result does not hold true. The problem is that this condition can be virtually impossible to verify in examples; a similar point was made in a recent work $[1$, p. 5].

The proofs of our criteria rely on Tauberian theorems and, as we expect, the monograph [5] is our splendid source. We then fine tune our machinery to the fashionable class of timechanged Lévy models [6] and [11] and see how it applies to the variance gamma model under the gamma-Ornstein-Uhlenbeck (gamma-OU) clock, the NIG model with Cox-Ingersoll-Ross (CIR) clock, and also the Heston model. The moral is that most, if not all, popular models for asset returns, with known MGF and $p^{*}, q^{*} \in(0, \infty)$, satisfy one of our criteria, so that (3) and (4) will hold. Numerical simulations demonstrate that the asymptotic regime becomes visible for remarkably low log strikes, which underlines the practical value of moment and tail-wing formulae.

\section{Background in regular variation}

For the reader's convenience, we collect some facts from the theory of regular variation. If $f=f(x)$ is defined and locally bounded on $[X, \infty)$ and tends to $\infty$ as $x$ tends to $\infty$ then the generalised inverse

$$
f^{\leftarrow}(x):=\inf \{y \in[X, \infty): f(y)>x\}
$$

is defined on $[f(X), \infty)$ and is monotone increasing to $\infty$. This applies, in particular, to $f \in R_{\alpha}$ with $\alpha>0$, and Thereom 1.5.12 of [5] asserts that $f \leftarrow \in R_{1 / \alpha}$ and

$$
f\left(f^{\leftarrow}(x)\right) \sim f^{\leftarrow}(f(x)) \sim x \quad \text { as } x \rightarrow \infty .
$$

Given $f$, we can often compute $f^{\leftarrow}$ (up to asymptotic equivalence) in terms of the Bruijn conjugate of slowly varying functions [5, Proposition 1.5.15]. A positive function $g$ defined in some neighbourhood of $\infty$ varies smoothly with index $\alpha, g \in S R_{\alpha}$, if and only if $h(x):=$ $\log \left(g\left(\mathrm{e}^{x}\right)\right)$ is $C^{\infty}$ and

$$
h^{\prime}(x) \rightarrow \alpha, \quad h^{(n)}(x) \rightarrow 0, \quad \text { for } n=2,3, \ldots \text { as } x \rightarrow \infty .
$$

The smooth variation theorem [5, Theorem 1.8.2] states that if $f \in R_{\alpha}$ then there exist $f_{i} \in S R_{\alpha}, i=1,2$, with $f_{1} \sim f_{2}$ and $f_{1} \leq f \leq f_{2}$ on some neighbourhood of $\infty$. When $\alpha>0$, we can assume that $f_{1}$ and $f_{2}$ are strictly increasing in some neighbourhood of $\infty$. In fact, we have the following result. 
Proposition 1. Let $\alpha>0$, and let $g \in S R_{\alpha}$. Then $g$ is strictly increasing in some neighbourhood of $\infty$ and $g^{\prime} \in S R_{\alpha-1}$.

Proof. By the definition of $S R_{\alpha}$,

$$
\frac{\partial}{\partial x} \log \left(g\left(\mathrm{e}^{x}\right)\right)=\frac{1}{g\left(\mathrm{e}^{x}\right)} g^{\prime}\left(\mathrm{e}^{x}\right) \mathrm{e}^{x} \rightarrow \alpha>0 \quad \text { as } x \rightarrow \infty .
$$

This shows that, in some neighbourhood of $\infty, g^{\prime}$ is strictly positive, which implies that $g$ is strictly increasing. From [5, Proposition 1.8.1], $g^{\prime}=\left|g^{\prime}\right| \in S R_{\alpha-1}$.

We remark that in the situation of Proposition 1 we have $\lim _{x \rightarrow \infty} g(x)=\infty$ and, hence, in some neighbourhood of $\infty, g$ has a genuine inverse $g^{-1}$ that coincides with the generalised inverse $g^{\leftarrow}$. Kohlbecker's Tauberian theorem [5, Theorem 4.1.2, Corollary 4.12.6] states that, for a nondecreasing, right-continuous function on $\mathbb{R}$ with $U(x)=0$ for all $x<0, \alpha>1$, and $\chi \in R_{\alpha /(\alpha-1)}$, we have

$$
\begin{aligned}
\log N(\lambda) & \sim \frac{(\alpha-1) \chi(\lambda)}{\lambda} \quad \text { as } \lambda \rightarrow \infty \\
\Longleftrightarrow \log \mu[0, x] & \sim \frac{\alpha x}{\chi \leftarrow(x)} \quad \text { as } x \rightarrow \infty,
\end{aligned}
$$

where $N(\lambda):=\int_{[0, \infty)} \mathrm{e}^{-x / \lambda} \mathrm{d} U(x), \lambda>0$. Karamata's Tauberian theorem [5, Theorem 1.7.1] states that if $l \in R_{0}, c \geq 0$, and $\rho \geq 0$ then

$$
\begin{gathered}
U(x) \sim \frac{c x^{\rho} l(x)}{\Gamma(1+\rho)} \quad \text { as } x \rightarrow \infty \\
\Longleftrightarrow \hat{U}(s) \equiv \int_{0}^{\infty} \mathrm{e}^{-s x} \mathrm{~d} U(x) \sim c s^{-\rho} l\left(\frac{1}{s}\right) \quad \text { as } s \rightarrow 0+.
\end{gathered}
$$

(When $c=0$, the asymptotic relations are interpreted in the sense that $U(x)=o\left(x^{\rho} l(x)\right.$ ), and similarly for $\hat{U}$.) Finally, Bingham's lemma [5, Theorem 4.12.10] states that, for $f \in R_{\alpha}$ with $\alpha>0$ such that $\mathrm{e}^{-f}$ is locally integrable at $+\infty$, we have the asymptotic relation

$$
-\log \int_{x}^{\infty} \mathrm{e}^{-f(y)} \mathrm{d} y \sim f(x) .
$$

\section{MGFs and $\log$ tails}

Let $F$ be a finite Borel measure on $\mathbb{R}$, identified with its (bounded, nondecreasing, rightcontinuous) distribution function, $F(x) \equiv F((-\infty, x])$. Its MGF is defined as

$$
M(s):=\int \mathrm{e}^{s x} \mathrm{~d} F(x) .
$$

We define the critical exponents $q^{*}$ and $r^{*}$ via

$$
-q^{*} \equiv \inf \{s: M(s)<\infty\}, \quad r^{*} \equiv \sup \{s: M(s)<\infty\},
$$

and make the standing assumption that

$$
r^{*}, q^{*} \in(0, \infty)
$$


In this section we develop criteria that will imply the asymptotic relations

$$
\log F((-\infty,-x]) \sim-q^{*} x, \quad \log F((x, \infty)) \sim-r^{*} x, \quad \text { as } x \rightarrow \infty .
$$

The assumption in Criterion 1, below, is simply that some derivative of the MGF (at the critical exponent) blows up in a regularly varying way.

Criterion 1. Let $F$ be a bounded, nondecreasing, right-continuous function on $\mathbb{R}$, and define $M=M(s), q^{*}$, and $r^{*}$ as above.

(i) If, for some $n \geq 0, M^{(n)}\left(-q^{*}+s\right) \sim s^{-\rho} l_{1}(1 / s)$ for some $\rho>0$ and $l_{1} \in R_{0}$ as $s \rightarrow 0+$ then

$$
\log F((-\infty,-x]) \sim-q^{*} x .
$$

(ii) If, for some $n \geq 0, M^{(n)}\left(r^{*}-s\right) \sim s^{-\rho} l_{1}(1 / s)$ for some $\rho>0$ and $l_{1} \in R_{0}$ as $s \rightarrow 0+$ then

$$
\log F((x, \infty)) \sim-r^{*} x .
$$

Proof. Let us focus on case (ii), noting that case (i) is similar. First we discuss $n=0$. The idea is an Escher-type change of measure followed by an application of Karamata's Tauberian theorem. We define a new measure $U$ on $[0, \infty)$ by a change-of-measure designed to remove the exponential decay,

$$
\mathrm{d} U(x):=\exp \left(r^{*} x\right) \mathrm{d} F(x) .
$$

We identify $U$ with its nondecreasing, right-continuous distribution function $x \mapsto U([0, x])$. The Laplace transform of $U$ is given by

$$
\begin{aligned}
\hat{U}(s) & =\int_{0}^{\infty} \mathrm{e}^{-s x} \mathrm{~d} U(x) \\
& =\int_{0}^{\infty} \exp \left(\left(r^{*}-s\right) x\right) \mathrm{d} F(x) \\
& =M\left(r^{*}-s\right)-\int_{-\infty}^{0} \exp \left(\left(r^{*}-s\right) x\right) \mathrm{d} F(x),
\end{aligned}
$$

so that

$$
\left|\hat{U}(s)-M\left(r^{*}-s\right)\right| \leq \int_{-\infty}^{0} \exp \left(\left(r^{*}-s\right) x\right) \mathrm{d} F(x) \leq F(0)-F(-\infty) \leq 2\|F\|_{\infty}<\infty .
$$

Since $M\left(r^{*}-s\right)$ goes to $\infty$ as $s \rightarrow 0+$ and we see that $\hat{U}(s) \sim M\left(r^{*}-s\right)$, then $\hat{U} \in R_{\rho}$ as $s \rightarrow 0$. Hence, there exists $l \in R_{0}$ such that $\hat{U}(s)=(1 / s)^{\rho} l(1 / s)$, and Karamata's Tauberian theorem tells us that $U \in R_{\rho}$, namely

$$
U(x) \sim x^{\rho} \frac{l(x)}{\Gamma(1+\rho)} \equiv x^{\rho} l^{\prime}(x) \quad \text { as } x \rightarrow \infty,
$$

where $l^{\prime} \in R_{0}$. Going back to the right tail of $F$, we have, for $x \geq 0$,

$$
F((x, \infty))=\int_{(x, \infty)} \mathrm{d} F(y)=\int_{(x, \infty)} \exp \left(-r^{*} y\right) \mathrm{d} U(y) .
$$


First we assume that $U \in S R_{\rho}$. Under this assumption, $U$ is smooth with derivative $u=U^{\prime} \in$ $S R_{\rho-1}$ and we can write

$$
u(y)=y^{\rho-1} l^{\prime \prime}(y) \quad \text { with } l^{\prime \prime} \in R_{0} .
$$

Then

$$
\begin{aligned}
F((x, \infty)) & =\int_{(x, \infty)} \exp \left(-r^{*} y\right) y^{\rho-1} l^{\prime \prime}(y) \mathrm{d} y \\
& =\int_{(x, \infty)} \exp \left(-r^{*} y+(\rho-1) \log y+\log l^{\prime \prime}(y)\right) \mathrm{d} y
\end{aligned}
$$

Since $-\left(-r^{*} y+(\rho-1) \log y+\log l^{\prime \prime}(y)\right) \sim r^{*} y \in R_{1}$ as $y \rightarrow \infty$, we can use Bingham's lemma to obtain

$$
-\log F((x, \infty))=-\log \int_{(x, \infty)} \exp \left(-r^{*} y+(\rho-1) \log y+\log l^{\prime \prime}(y)\right) \mathrm{d} U(y) \sim r^{*} y .
$$

Now we deal with the general case of nondecreasing $U \in R_{\rho}$. From the smooth variation theorem and Proposition 1, we can find $U_{-}, U_{+} \in S R_{\rho}$ strictly increasing in a neighbourhood of $\infty$, so that

$$
U_{-} \leq U \leq U_{+} \quad \text { and } \quad U_{-} \sim U \sim U_{+}
$$

Below we use the change of variables $z=U(y)$ and $w=U_{+}^{-1}(z)$. Noting that $U_{+}^{-1} \leq U^{\leftarrow} \leq$ $U_{-}^{-1}$ and using the change-of-variable formulae, as found in [10, pp. 7-9] for instance, we have

$$
\begin{aligned}
F((x, \infty)) & =\int_{(x, \infty)} \exp \left(-r^{*} y\right) \mathrm{d} U(y) \\
& =\int_{(U(x), \infty)} \exp \left(-r^{*} U^{\leftarrow}(z)\right) \mathrm{d} z \\
& \leq \int_{(U(x), \infty)} \exp \left(-r^{*} U_{+}^{-1}(z)\right) \mathrm{d} z \\
& =\int_{\left(U_{+}^{-1}(U(x)), \infty\right)} \exp \left(-r^{*} w\right) \mathrm{d} U_{+}(w) .
\end{aligned}
$$

Similar to the derivation of (6), Bingham's lemma leads to

$$
-\log \int_{\left(U_{+}^{-1}(U(x)), \infty\right)} \exp \left(-r^{*} w\right) \mathrm{d} U_{+}(w) \sim r^{*} U_{+}^{-1}(U(x)) .
$$

Noting that $U_{+}^{-1}$ is nondecreasing, $U_{+}^{-1}(U(x)) \leq U_{+}^{-1}\left(U_{+}(x)\right)=x$ so that

$$
-\log F([x, \infty)) \lesssim r^{*} x
$$

where by $g \lesssim h$ we mean $\lim \sup f(x) / g(x) \leq 1$ as $x \rightarrow \infty$. The same argument gives the lower bound $-\log F((x, \infty)) \gtrsim r^{*} x$, and we conclude that

$$
-\log F((x, \infty)) \sim r^{*} x .
$$

Now we show how $n>0$ follows from $n=0$. Define $V$ on $[0, \infty)$ by

$$
\mathrm{d} V(x):=x^{n} \mathrm{~d} F(x) .
$$


Clearly, $V$ induces a nondecreasing, right-continuous distribution on $\mathbb{R}, V(x):=V([0, x])$ for $x \geq 0$ and $V(x) \equiv 0$ for $x<0$. The distribution function $V(x)$ is also bounded since

$$
\int_{0}^{\infty} x^{n} \mathrm{~d} F(x)<\infty
$$

which follows a forteriori from the standing assumption of exponential moments. We will write $\bar{V}(x)$ for $V(x, \infty)$.

Note that $V$ has an MGF $M_{V}(s)$, finite at least for $s \in\left(0, r^{*}\right)$, given by

$$
\begin{aligned}
M_{V}(s) & \equiv \int \mathrm{e}^{s x} \mathrm{~d} V(x) \\
& =\int_{0}^{\infty} x^{n} \mathrm{e}^{s x} \mathrm{~d} F \\
& =\int x^{n} \mathrm{e}^{s x} \mathrm{~d} F+C \\
& =M^{(n)}(s)+C,
\end{aligned}
$$

where

$$
0 \leq C \equiv-\int_{-\infty}^{0} x^{n} \mathrm{e}^{s x} \mathrm{~d} F \leq \int_{-\infty}^{0}|x|^{n} \mathrm{~d} F<\infty .
$$

(We could do without the assumption $\int_{-\infty}^{0}|x| \mathrm{d} F$, which follows a forteriori from the standing assumption $q^{*}>0$. Finiteness of $F$ on $(-\infty, 0)$ is enough.) By assumption, $M^{(n)}$ is regularly varying with index $\rho$ at $r^{*}$ and it follows that, as $s \rightarrow 0+$,

$$
M_{V}\left(r^{*}-s\right)=M^{(n)}\left(r^{*}-s\right)+O(1) \sim s^{-\rho} l_{1}\left(\frac{1}{s}\right) .
$$

Now we use the ' $n=0$ ' result on the distribution function $V$, respectively its MGF $M_{V}$, and obtain

$$
-\log V([x, \infty)) \equiv-\log \bar{V}(x) \sim r^{*} x \in R_{1} .
$$

First assume that $-\log \bar{V}(x) \in S R_{1}$. Then $V$ has a density $V^{\prime} \equiv v$ and

$$
v(x)=\partial_{x}(V(\infty)-\bar{V}(x))=-\bar{V}(x) \partial_{x}(\log \bar{V}(x)) \sim r^{*} \bar{V}(x) \quad \text { as } x \rightarrow \infty,
$$

since functions in $S R_{1}$ are stable under differentiation in the sense that $\partial_{x}(-\log \bar{V}(x)) \sim$ $\partial_{x}\left(r^{*} x\right)=r^{*}$. In particular, we have $\log v(x) \sim \log \bar{V}(x) \sim-r^{*} x$. After these preparations, we can write

$$
\begin{aligned}
F((x, \infty)) & =\int_{(x, \infty)} \mathrm{d} F(y) \\
& =\int_{(x, \infty)} \frac{1}{y^{n}} v(y) \mathrm{d} y \\
& =\int_{(x, \infty)} \exp (\log v(y)-n \log y) \mathrm{d} y,
\end{aligned}
$$

and Bingham's lemma implies that $\log F((x, \infty)) \sim-r^{*} x$. The general case of $\log \bar{V}(x) \in R_{1}$ follows by a smooth variation and comparison argument, as earlier. 
Criterion 2, below, deals with the exponential blow up of $M$ at its critical values.

Criterion 2. Let $F, M, q^{*}$, and $r^{*}$ be as defined above.

(i) If $\log M\left(-q^{*}+s\right) \sim s^{-\rho} l_{1}(1 / s)$ for some $\rho>0$ and $l_{1} \in R_{0}$ as $s \rightarrow 0+$ then

$$
\log F((-\infty,-x]) \sim-q^{*} x .
$$

(ii) If $\log M\left(r^{*}-s\right) \sim s^{-\rho} l_{1}(1 / s)$ for some $\rho>0$ and $l_{1} \in R_{0}$ as $s \rightarrow 0+$ then

$$
\log F((x, \infty)) \sim-r^{*} x .
$$

Proof. As for Criterion 1, the idea is an Escher-type change of measure followed by a suitable Tauberian theorem; in the present case we need Kohlbecker's theorem. Let us focus on case (ii), noting that case (i) is similar. A new measure $U$ on $[0, \infty)$ is defined by

$$
\mathrm{d} U(x):=\exp \left(r^{*} x\right) \mathrm{d} F(x) .
$$

We identify $U$ with its nondecreasing, right-continuous distribution function $x \mapsto U([0, x])$, and define the transform

$$
\begin{aligned}
N(\lambda) & =\int_{0}^{\infty} \mathrm{e}^{-x / \lambda} \mathrm{d} U(x) \\
& =\int_{0}^{\infty} \exp \left(\left(r^{*}-\frac{1}{\lambda}\right) x\right) \mathrm{d} F(x) \\
& =M\left(r^{*}-\frac{1}{\lambda}\right)-\int_{-\infty}^{0} \exp \left(\left(r^{*}-\frac{1}{\lambda}\right) x\right) \mathrm{d} F(x),
\end{aligned}
$$

so that

$$
\left|N(\lambda)-M\left(r^{*}-\frac{1}{\lambda}\right)\right| \leq \int_{-\infty}^{0} \exp \left(\left(r^{*}-\frac{1}{\lambda}\right) x\right) \mathrm{d} F(x) \leq F(0)-F(-\infty) \leq 2\|F\|_{\infty}<\infty .
$$

Thus,

$$
N(\lambda)=M\left(r^{*}-\frac{1}{\lambda}\right)+O(1) \quad \text { as } \lambda \rightarrow \infty,
$$

and, in particular, since

$$
\lim _{\lambda \rightarrow \infty} \log M\left(r^{*}-\frac{1}{\lambda}\right)=\lim _{\lambda \rightarrow \infty} M\left(r^{*}-\frac{1}{\lambda}\right)=\infty
$$

from assumption (ii), we see that

$$
\log N(\lambda) \sim \log M\left(r^{*}-\frac{1}{\lambda}\right) \sim \lambda^{\rho} l_{1}(\lambda) \in R_{\rho} \quad \text { as } \lambda \rightarrow \infty .
$$

Define $\alpha \in(1, \infty)$ as the unique solution to $\rho+1=\alpha /(\alpha-1)$, and note that

$$
\chi(\lambda):=\frac{\lambda}{\alpha-1} \log N(\lambda) \in R_{\rho+1}=R_{\alpha /(\alpha-1)} .
$$


Using the fact that $\chi^{\leftarrow} \in R_{(\alpha-1) / \alpha}=R_{1-1 / \alpha}$, Kohlbecker's Tauberian theorem tells us that

$$
\log U([0, x]) \equiv \log U(x) \sim \frac{\alpha x}{\chi^{\leftarrow}(x)} \in R_{1 / \alpha} \quad \text { as } x \rightarrow \infty
$$

In particular, there exists $l \in R_{0}$ such that $\log U(x)=\alpha x^{1 / \alpha} l(x)$. First we assume that $\log U \in S R_{1 / \alpha}$. Then $U$ has a density $u(\cdot) \in S R_{1 / \alpha-1}$ and

$$
u(x)=U(x) \partial_{x}(\log U(x)) \sim U(x) x^{1 / \alpha-1} l(x) .
$$

In particular,

$$
\log u(x) \sim \log U(x) \in R_{1 / \alpha} \quad \text { as } x \rightarrow \infty .
$$

Now, $y \mapsto r^{*} y \in R_{1}$ dominates $R_{1 / \alpha}$ (since $1 / \alpha<1$ ) in the sense that

$$
r^{*} y-\log u(y) \sim r^{*} y .
$$

Thus, from

$$
\begin{aligned}
F((x, \infty)) & =\int_{(x, \infty)} \mathrm{d} F(y) \\
& =\int_{[x, \infty)} \exp \left(-r^{*} y\right) u(y) \mathrm{d} y \\
& =\int_{(x, \infty)} \exp \left(-r^{*} y+\log u(y)\right) \mathrm{d} y
\end{aligned}
$$

and Bingham's lemma, we deduce that

$$
-\log F((x, \infty)) \sim r^{*} x .
$$

The general case, $\log U \in R_{1 / \alpha}$, is handled via smooth variation, as earlier. Namely, we can find smooth minorising and majorising functions for $\log U$, say $G_{-}$and $G_{+}$, respectively. After defining $U_{ \pm}=\exp G_{ \pm}$, we have

$$
\log U_{-} \sim \log U \sim \log U_{+} \quad \text { and } \quad U_{-} \leq U \leq U_{+} .
$$

Then, exactly as in the last step of the proof of Criterion 1,

$$
F((x, \infty))=\int_{(x, \infty)} \exp \left(-r^{*} y\right) \mathrm{d} U(y) \leq \int_{\left(U_{+}^{-1}(U(x)), \infty\right)} \exp \left(-r^{*} w\right) \mathrm{d} U_{+}(w),
$$

and, from Bingham's lemma,

$$
-\log F((x, \infty)) \lesssim r^{*} U_{+}^{-1}(U(x)) \sim r^{*} x .
$$

Similarly, $-\log F((x, \infty)) \gtrsim r^{*} x$ and the proof is complete.

\section{Application to smile asymptotics}

We start with a few recalls to settle the notation. The normalised Black-Scholes formula for a call with $\log$ strike $k$ is given by

$$
c_{\mathrm{BS}}(k, \sigma)=\Phi\left(d_{1}\right)-\mathrm{e}^{k} \Phi\left(d_{2}\right),
$$


where $\Phi$ is the cumulative distribution function of a standard Gaussian, with $d_{1,2}(k)=-k / \sigma \pm$ $\sigma / 2$. If we model risk-neutral returns with a distribution function $F$, the implied volatility is the (unique) value $V(k)$ so that

$$
c_{\mathrm{BS}}(k, V(k))=\int_{k}^{\infty}\left(\mathrm{e}^{x}-\mathrm{e}^{k}\right) \mathrm{d} F(x)=: c(k) .
$$

Set $\psi[x] \equiv 2-4\left(\sqrt{x^{2}+x}-x\right)$, and recall that $\bar{F} \equiv 1-F$. The following is a special case of the tail-wing formula [4].

Theorem 1. Assume that $-\log F(-k) / k \sim q^{*}$ for some $q^{*} \in(0, \infty)$. Then

$$
\frac{V(-k)^{2}}{k} \sim \psi\left(-\frac{\log F(-k)}{k}\right) \sim \psi\left(q^{*}\right) .
$$

Similarly, assume that $-\log \bar{F}(k) / k \sim p^{*}+1$ for some $p^{*} \in(0, \infty)$. Then

$$
\frac{V(k)^{2}}{k} \sim \psi\left(-1-\frac{\log \bar{F}(k)}{k}\right) \sim \psi\left(p^{*}\right) .
$$

As earlier, let $M(s)=\int \mathrm{e}^{s x} \mathrm{~d} F(x)$ denote the MGF of risk-neutral returns, and now define the critical exponents $r^{*}$ and $-q^{*}$ exactly as in the beginning of Section 3. Combining the results therein with Theorem 1, we obtain the following result.

Theorem 2. If $q^{*} \in(0, \infty)$ and $M$ satisfies part (i) of Criteria 1 or 2 then

$$
\frac{V(-k)^{2}}{k} \sim \psi\left(q^{*}\right) \quad \text { as } k \rightarrow \infty .
$$

Similarly, if $r^{*} \equiv p^{*}+1 \in(1, \infty)$ and $M$ satisfies part (ii) of Criteria 1 or 2 then

$$
\frac{V(k)^{2}}{k} \sim \psi\left(p^{*}\right) \text { as } k \rightarrow \infty .
$$

\section{First examples}

We discuss several exponential Lévy models; the risk-neutral log price is thus given by a genuine Lévy process. For models that satisfy one of our criteria, the asymptotic slope of the total implied variance (i.e. the implied Black-Scholes volatility squared times the maturity $T$ ) is determined by the critical exponents in the MGF and by the Lévy-Khinchine formula, this value will not depend on maturity. (This time-invariance property must not be confused with the floating smile property of exponential Lévy models, e.g. Proposition 11.1 of [6], which is a statement about the evolution of implied volatility over time: the implied volatility for maturity $T+t$, as seen from time $t$, is independent of $t$ provided that the implied volatility is parametrised in terms of the moneyness and remaining time to maturity. In contrast, all the results of this paper are concerned with the implied volatility (respectively variance) at time $T$ as seen from time $t=0$.)

\subsection{Criterion 1 with $\boldsymbol{n}=0$ : the variance gamma model}

The variance gamma model $\mathrm{VG}=\mathrm{VG}(m, g, C)$ has MGF given by

$$
M(s)=\left(\frac{g m}{g m+(m-g) s-s^{2}}\right)^{C}=\left(\frac{g m}{(m-s)(s+g)}\right)^{C} .
$$


The critical exponents are obviously given by $r^{*}=m$ and $q^{*}=g$. Focusing on the first, we have

$$
M\left(r^{*}-s\right) \sim\left(\frac{g m}{m+g}\right)^{C} s^{-C} \quad \text { as } s \rightarrow 0+,
$$

which shows that Criterion 1 is satisfied with $n=0$. Theorem 2 now identifies the asymptotic slope of the implied variance to be $\psi\left(r^{*}-1\right)=\psi(m-1)$. Similarly, the left slope is seen to be $\psi\left(q^{*}\right)=\psi(g)$. We remark that [1] contains tail estimates for VG that lead, via the tail-wing formula, to the same result.

\subsection{Criterion 1 with $\boldsymbol{n}>0$ : the NIG model}

The NIG model NIG $=$ NIG $(\alpha, \beta, \mu, \delta)$ has MGF given by

$$
M(s)=\exp \left(\delta\left(\sqrt{\alpha^{2}-\beta^{2}}-\sqrt{\alpha^{2}-(\beta+s)^{2}}\right)+\mu s\right) .
$$

By looking at the endpoints of the strip of analyticity, the critical exponents are immediately seen to be $r^{*}=\alpha-\beta$ and $q^{*}=\alpha+\beta$, and we focus again on the first. While $M(s)$ converges to the finite constant $M\left(r^{*}\right)$ as $s \rightarrow r^{*}-$, we have

$$
\frac{M^{\prime}(s)}{M(s)}=\left(2 \delta(\beta+s)\left(\alpha^{2}-(\beta+s)^{2}\right)^{-1 / 2}+\mu\right)
$$

and

$$
M^{\prime}\left(r^{*}-s\right) \sim 2 \delta \alpha \sqrt{2 \alpha} s^{-1 / 2} M\left(r^{*}\right) \quad \text { as } s \rightarrow 0+.
$$

We see that Criterion 1 is satisfied with $n=1$, and Theorem 2 gives the asymptotic slope $\psi\left(r^{*}-1\right)=\psi(\alpha-\beta-1)$. Similarly, the left slope is seen to be $\psi\left(q^{*}\right)=\psi(\alpha+\beta)$. We remark that the same slopes were computed in [4] using the tail-wing formula and explicitly known density asymptotics for NIG.

\subsection{Criterion 2: the double exponential model}

The double exponential model $\mathrm{DE}=\mathrm{DE}\left(\sigma, \mu, \lambda, p, q, \eta_{1}, \eta_{2}\right)$ has MGF given by

$$
\log M(s)=\frac{1}{2} \sigma^{2} s^{2}+\mu s+\lambda\left(\frac{p \eta_{1}}{\eta_{1}-s}+\frac{q \eta_{2}}{\eta_{2}+s}-1\right) .
$$

Clearly, $r^{*}=\eta_{1}$ and, as $s \rightarrow 0+$,

$$
\log M\left(\eta_{1}-s\right) \sim \frac{1}{2} \sigma \eta_{1}^{2}+\mu \eta_{1}+\lambda\left(\frac{p \eta_{1}}{s}+\frac{q \eta_{2}}{\eta_{2}+\eta_{1}}-1\right) \sim \lambda p \eta_{1} s^{-1},
$$

and we see that Criterion 2 is satisfied. As above, this implies asymptotic slopes $\psi\left(r^{*}-1\right)=$ $\psi\left(\eta_{1}-1\right)$ on the right and $\psi\left(\eta_{2}\right)$ on the left.

\section{Time-changed Lévy processes}

We now discuss how to apply our results to time-changed Lévy processes [6], [11], [12]. To do this, we only need to check that the MGF of the marginals of the process satisfies one of our criteria.

To this end, consider a Lévy process $L=L(t)$ described through its cumulant generating function (CGF) $K_{L}$ at time 1 , that is,

$$
K_{L}(v)=\log \mathrm{E}\left[\exp \left(v L_{1}\right)\right],
$$


and an independent random clock $T=T(\omega) \geq 0$ with CGF $K_{T}$. It follows that the MGF of $L \circ T$ is given by

$$
M(v)=\mathrm{E}\left[\mathrm{E}\left(\exp \left(v L_{T}\right) \mid T\right)\right]=\mathrm{E}\left[\exp \left(K_{L}(v) T\right)\right]=\exp \left(K_{T}\left(K_{L}(v)\right)\right) .
$$

Therefore, in order to apply our Theorem 2 to time-changed Lévy models, we need to check if $M=\exp \left(K_{T}\left(K_{L}(\cdot)\right)\right)$ satisfies Criteria 1 or 2 then $-\log \bar{F}(x) / x$ tends to a positive constant. Here, as earlier, $F$ denotes the distribution function of $M$ and $\bar{F} \equiv 1-F$. Theorem 3, below, gives sufficient conditions for this in terms of $K_{T}$ and $K_{L}$. We shall write

$$
M_{T} \equiv \exp \left(K_{T}\right) \quad \text { and } \quad M_{L} \equiv \exp \left(K_{L}\right)
$$

For brevity, we only discuss the right tail (in fact, the elegant change-of-measure argument in [8] allows a formal reduction of the left-tail behaviour to the right-tail behaviour) and set

$$
p_{L}=\sup \left\{s: M_{L}(s)<\infty\right\} \quad \text { and } \quad p_{T}=\sup \left\{s: M_{T}(s)<\infty\right\} .
$$

Theorem 3. Using the same notation as above and assuming that $p_{L}, p_{T}>0$, we have the following.

(i) If $K_{L}(p)=p_{T}$ for some $p \in\left[0, p_{L}\right)$ and $M_{T}$ satisfies either Criteria 1 or 2 then

$$
\log \bar{F}(x) \sim-p x .
$$

(ii) If $K_{L}(p)=p_{T}$ for $p=p_{L}$ and $M_{T}$ and $M_{L}$ satisfy either Criteria 1 or 2 then

$$
\log \bar{F}(x) \sim-p x .
$$

(iii) If $K_{L}(p)<p_{T}$ for all $p \in\left[0, p_{L}\right]$ and $M_{L}$ satisfies either Criteria 1 or 2 then

$$
\log \bar{F}(x) \sim-p_{L} x .
$$

Remark 1. It is worth noting that there cannot be more than one solution to $K_{L}(p)=p_{T}$. To see this, take any $v$ such that $v>0$ and $K_{L}(v)>0$ (any solution to $K_{L}(p)=p_{T}>0$ will satisfy this!). From $M_{L} \equiv \exp \left(K_{L}\right)$, it follows that

$$
M_{L}(0)=1 \quad \text { and } \quad M_{L}(v)>1 .
$$

From this and the convexity of $M_{L}(\cdot)$, it is easy to see that $M_{L}^{\prime}(v)$ is strictly positive and the same is true for $K_{L}^{\prime}(v)=M_{L}^{\prime}(v) / M_{L}(v)$. It follows that $w \geq v$ implies that $K_{L}(w) \geq K_{L}(v)>0$, the set of all $\left\{v>0: K_{L}(v)>0\right\}$ is connected, and that $K_{L}$ restricted to this set is strictly increasing. This shows that there is at most one solution to $K_{L}(p)=p_{T}$.

Proof of Theorem 3. (i) Noting that $p>0$, let us first assume that $M_{T}$ satisfies Criterion 1 (at $K_{L}(p)=p_{T}$ with some $n \geq 0$ ), so that, for some $\rho>0$ and $l \in R_{0}$,

$$
M_{T}^{(n)}(u) \sim\left(p_{T}-u\right)^{-\rho} l\left(\left(p_{T}-u\right)^{-1}\right) \quad \text { as } u \uparrow p_{T} .
$$

From $M=M_{T} \circ K_{L}$ we have

$$
M^{\prime}=M_{T}^{\prime}\left(K_{L}\right) K_{L}^{\prime}
$$


and, by iteration, $M^{(n)}$ equals $M_{T}^{(n)}\left(K_{L}\right)\left(K_{L}^{\prime}\right)^{n}$ plus a polynomial in $M_{T}(\cdot), \ldots, M_{T}^{(n-1)}(\cdot)$ which remains bounded when the argument approaches $p_{T}$. Noting that $K_{L}^{\prime}(p)>0$ (see Remark 1), we absorb the factor $\left(K_{L}^{\prime}(p)\right)^{n}$ into the slowly varying function and see that

$$
M^{(n)}(v) \sim\left(p_{T}-K_{L}(v)\right)^{-\rho} l\left(\left(p_{T}-K_{L}(v)\right)^{-1}\right) \quad \text { for } \rho \text { as above and some } l \in R_{0}
$$

as $K_{L}(v)$ tends to $p_{T}$, which follows from $v \uparrow p$. Using analyticity of $K_{L}$ in $\left(0, p_{L}\right)$ and $K_{L}^{\prime}(p) \neq 0$, it is clear that

$$
p_{T}-K_{L}(v) \sim K_{L}^{\prime}(p)(p-v) \quad \text { as } v \uparrow p,
$$

and so

$$
M^{(n)}(p-v) \sim K_{L}^{\prime}(p)^{-\rho} v^{-\rho} l\left(\frac{1}{v}\right) \quad \text { as } v \rightarrow 0+.
$$

This shows that $M$ satisfies Criterion 1 (with the same $n$ as $M_{T}$ ). A similar argument shows that $M$ satisfies Criterion 2 if $M_{T}$ does. Either way, the asserted tail behaviour of $\log \bar{F}$ follows.

(ii) The (unlikely!) case in which $K_{L}\left(p_{L}\right)=p_{T}$ involves similar ideas and is left to the reader.

(iii) Now we assume that $\sup _{p \in\left[0, p_{L}\right]} K_{L}(p)<p_{T}<\infty$, and that $M_{L}$ satisfies either Criteria 1 or 2 (at $p_{L}$ ). Since $M_{L}=\exp \left(K_{L}\right)$ stays bounded as its argument approaches the critical value $p_{L}$, it is clear that $M_{L}$ cannot satisfy Criteria 1 or 2 with $n=0$ and so there must exist a smallest integer $n$ such that $M_{L}^{(n)}\left(p_{L}-x\right) \sim x^{-\rho} l(x)$ as $x \rightarrow p_{L}$ for some $\rho>0$ and $l \in R_{0}$. We note that

$$
M^{(n)}(v)=\left(K_{L}^{(n)}(v) K_{T}^{\prime}\left(K_{L}(v)\right)+f(v)\right) \exp \left(K_{T}\left(K_{L}(v)\right)\right),
$$

where $f(v)$ is a polynomial function of the first $(n-1)$ derivatives of $K_{L}$ and the first $n$ derivatives of $K_{T}$ evaluated at $K_{L}(v)$, which are all bounded for $0 \leq v \leq p_{L}$. Noting that the positivity of $T$ implies that $M_{T}^{\prime}>0$ and, hence, $K_{T}^{\prime}>0$, we see that, as $v \uparrow p_{L}$,

$$
M^{(n)}(v) \sim K_{L}^{(n)}(v) K_{T}^{\prime}\left(K_{L}\left(p_{L}\right)\right) M\left(p_{L}\right) .
$$

Applying this to $K_{T}(x) \equiv x$ leads immediately to

$$
K_{L}^{(n)}(v) \sim \frac{M_{L}^{(n)}(v)}{M_{L}(v)} \sim \frac{x^{-\rho} l(x)}{M_{L}\left(p_{L}\right)}
$$

as $v \uparrow p_{L}$, and so $M$ satisfies Criterion 1 .

Now we discuss examples to which the above analysis is applicable. For all the examples, we plot the total variance smile (that is, $\left.V^{2}(k, t) \equiv \sigma^{2}(k, t) t\right)$ for several maturities and compare with straight lines, which have been parallel shifted so that they are easier to compare with the actual smile, that have correct slope predicted using Theorem 2. All the plots are based on parameters fitted to market data in [12].

\subsection{The VG process with gamma-OU time change}

We will consider the VG process with a gamma-OU time change and refer to [12] for details. From earlier, the VG process has CGF given by

$$
K_{L}(v)=C \log \left(\frac{g m}{(m-v)(v+g)}\right) \text { for } v \in(-g, m) .
$$


We note that $K_{L}([0, m])=[0, \infty]$, so that $p_{L}=m$. The gamma-OU clock $T=T(\omega, t)$ has CGF given by

$$
K_{T}(v)=v y_{0} \lambda^{-1}\left(1-\mathrm{e}^{-\lambda t}\right)+\frac{\lambda a}{v-\lambda b}\left(b \log \left(\frac{b}{b-v \lambda^{-1}\left(1-\mathrm{e}^{-\lambda t}\right)}\right)-v t\right) .
$$

We need to examine how this function behaves around the endpoint of its strip of regularity. At first glance, it appears that the function tends to $\infty$ as $v \uparrow \lambda b$, because of the $\lambda a /(v-\lambda b)$ term. However, upon closer examination, we can see that this is in fact a removable singularity and the term of interest to us is the $\log (\cdot)$ term. This term tends to $\infty$ as $v \rightarrow \lambda b\left(1-\mathrm{e}^{-\lambda t}\right)^{-1}=: p_{T}$. After some simple algebra, we see that

$$
\begin{aligned}
\exp \left(K_{T}(v)\right) & =\left(\frac{b}{b-v \lambda^{-1}\left(1-\mathrm{e}^{-\lambda t}\right)}\right)^{\lambda a b /(v-\lambda b)} \exp \left(v y_{0} \lambda^{-1}\left(1-\mathrm{e}^{-\lambda t}\right)-\frac{v t \lambda a}{v-\lambda b}\right) \\
& \sim\left(\frac{p_{T}}{p_{T}-v}\right)^{\lambda a b /\left(p_{T}-\lambda b\right)} \exp \left(p_{T} y_{0} \lambda^{-1}\left(1-\mathrm{e}^{-\lambda t}\right)-\frac{p_{T} t \lambda a}{p_{T}-\lambda b}\right) \quad \text { as } v \uparrow p_{T} .
\end{aligned}
$$

Therefore, $\exp \left(K_{T}\right)$ satisfies Criterion 1 with $n=0$ and Theorem 3(i) shows that $M$ satisfies Criterion 1 also. So, $\log \bar{F}(x) \sim-p x$, where $p$ is determined by the equation

$$
K_{L}(p)=p_{T}=\lambda b\left(1-\mathrm{e}^{-\lambda t}\right)^{-1}
$$

and can be calculated explicitly as

$$
p=\frac{m-g+\sqrt{(m-g)^{2}+4 g m\left(1-\exp \left(-\lambda b / C\left(1-\mathrm{e}^{-\lambda t}\right)\right)\right)}}{2} .
$$

See Figure 1 for a numerical example.

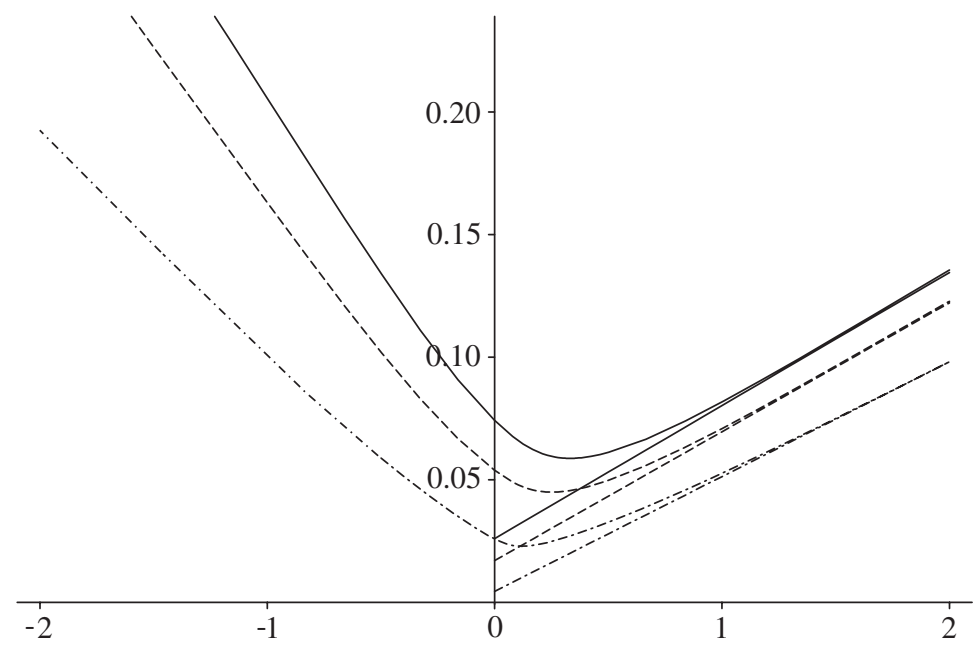

FIgURE 1: The VG process with gamma-OU time change. Parameters from [12]. The total implied variance and slopes for three maturities $t=0.4$ (dash-dot line), 0.9 (dashed line), and 1.3 (solid line) years. 


\subsection{The NIG model with CIR time change}

The CGF of the CIR clock $T=T(\omega, t)$ is given by

$$
K_{T}(v)=\frac{\kappa^{2} \eta t}{\lambda^{2}}+\frac{2 y_{0} v}{\kappa+\gamma \operatorname{coth}(\gamma t / 2)}-\frac{2 \kappa \eta}{\lambda^{2}} \log \left[\sinh \frac{\gamma t}{2}\left(\operatorname{coth} \frac{\gamma t}{2}+\frac{\kappa}{\gamma}\right)\right],
$$

where

$$
\gamma=\sqrt{\kappa^{2}-2 \lambda^{2} v}
$$

This clearly tends to $\infty$ as

$$
I(v) \equiv \kappa+\gamma(v) \operatorname{coth} \frac{\gamma(v) t}{2} \rightarrow 0,
$$

and we can define $p_{T}$ as a solution to the equation $I\left(p_{T}\right)=0$. Using l'Hôpital's rule, it is easy to check that

$$
\frac{p_{T}-v}{\kappa+\gamma(v) \operatorname{coth}(\gamma(v) t / 2)} t
$$

tends to a constant as $v \rightarrow p_{T}$, and so

$$
\frac{2 y_{0} v}{\kappa+\gamma \operatorname{coth}(\gamma t / 2)}
$$

is regularly varying of index 1 as a function of $\left(p_{T}-v\right)^{-1}$. It is clear that this is the dominant term in this limit, and so $M_{T} \equiv \exp \left(K_{T}\right)$ satisfies Criterion 2 (at $p_{T}$ ). From earlier, the NIG CGF is (following [12] we take $\mu=0$ here)

$$
K_{L}(v)=-\delta\left(\sqrt{\alpha^{2}-(\beta+v)^{2}}-\sqrt{\alpha^{2}-\beta^{2}}\right) \text { for } v \leq \alpha-\beta,
$$

from which we see that $p_{L}=\alpha-\beta>0$ and

$$
\sup _{v \in[0, \alpha-\beta]} K_{L}(v)=\delta \sqrt{\alpha^{2}-\beta^{2}} .
$$

Therefore, the behaviour of $M$ on the edge of the strip of analyticity, and the location of the critical value, will depend on whether this supremum is more or less than $p_{T}$; if it is less than $p_{T}$, the latter is never reached. Recalling that $\exp \left(K_{L}\right)$ satisfies Criterion 1 with $n=1$, we apply Theorem 3(ii) and obtain

$$
-\log \bar{F}(x) \sim p_{L} x=(\alpha-\beta) x .
$$

Otherwise, there exists $p \in(0, \alpha-\beta]$ such that $K_{L}(p)=p_{T}$ for some $p \leq \alpha-\beta$, and since $M_{T}$ was seen to satisfy one of the criteria (to be precise, Criterion 2), we can apply Theorem 3(i) and (ii) to obtain $-\log \bar{F}(x) \sim p x$. In particular, we see that, for all possible parameters in the NIG-CIR model, (2) holds true. Smile asymptotics are now an immediate consequence of Theorem 1. See Figure 2 for a numerical example. 


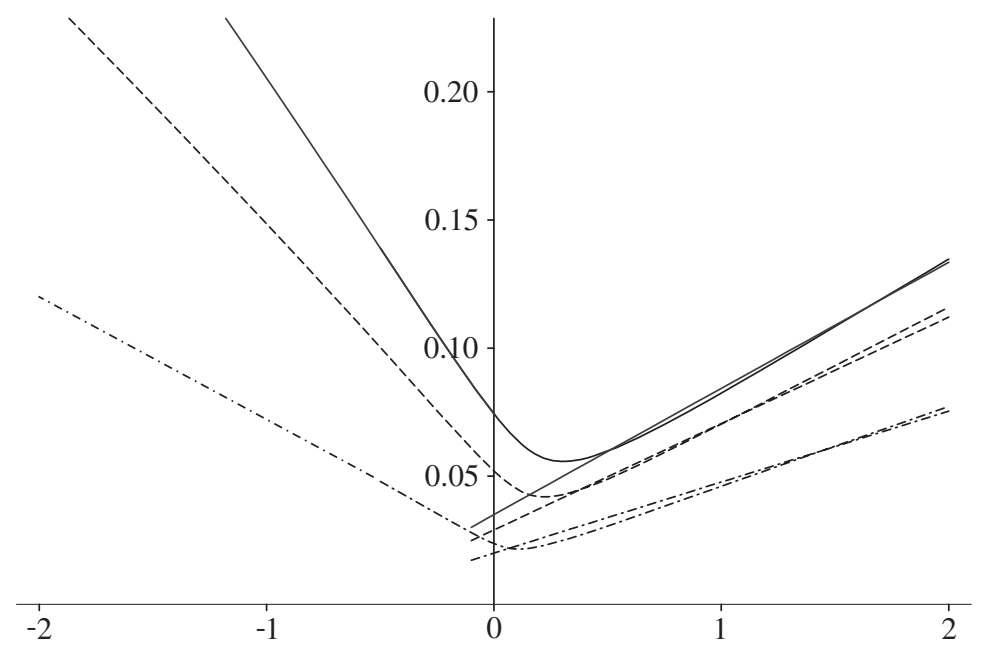

FIgURE 2: The NIG model with CIR time change. Parameters from [12]. The total implied variance and slopes for three maturities $t=0.4$ (dash-dot line), 0.9 (dashed line), and 1.3 (solid line) years. Observe that the lines with correct slope do not perfectly line up with the smile, which is not a contradiction to the result that $V^{2}(k) / k$ converges to a constant.

\subsection{The Heston model}

The Heston model is a stochastic volatility model defined by the following stochastic differential equations:

$$
\begin{aligned}
\frac{\mathrm{d} S_{t}}{S_{t}} & =\sqrt{v_{t}} \mathrm{~d} W_{t}^{1}, \\
\mathrm{~d} v_{t} & =\kappa\left(\eta-v_{t}\right) \mathrm{d} t+v_{t} \mathrm{~d} W_{t}^{2},
\end{aligned}
$$

where $\mathrm{d}\left\langle W_{t}^{1}, W_{t}^{2}\right\rangle=\rho \mathrm{d} t$ is the correlation of the two Brownian motions. Therefore, $\log S_{t}$ has the distribution of a Brownian motion with drift $-\frac{1}{2}$ evaluated at a random time $T(\omega, t)=$ $\int_{0}^{t} v_{s} \mathrm{~d} s$ with the distribution of an integrated CIR process, as in the previous example. When $\rho=0$, the Lévy process $L \equiv W^{1}$ and $T$ are independent and we can apply the same analysis as above. Namely, the CGF of the Brownian motion with drift speed $-\frac{1}{2}$ at time 1 is

$$
K_{L}(v)=\frac{v^{2}-v}{2}
$$

so that $p_{L}=\infty$, and $M_{T}=\exp \left(K_{T}\right)$ satisfies Criterion 2; hence, by Theorem 3(i) and (ii),

$$
\log \bar{F}(x) \sim-p x,
$$

where $p$ is determined by the equation $K_{L}(p)=p_{T}$. When $\rho \leq 0$, we can analyse the MGF of $\log S_{t}$ directly and apply the same reasoning as for the MGF of the CIR process to deduce that Criterion 2 is satisfied. Hence, the distribution function for the Heston returns satisfies $\log \bar{F}(x) \sim-p x$, where $p$ is solution to (see [2])

$$
(\kappa-\rho v \theta)+\left.\left(\theta^{2}\left(v^{2}-v\right)-(\kappa-\rho v \theta)^{2}\right)^{1 / 2} \cot \left(\frac{\left(\theta^{2}\left(v^{2}-v\right)-(\kappa-\rho v \theta)^{2}\right)^{1 / 2} t}{2}\right)\right|_{v=p}=0
$$




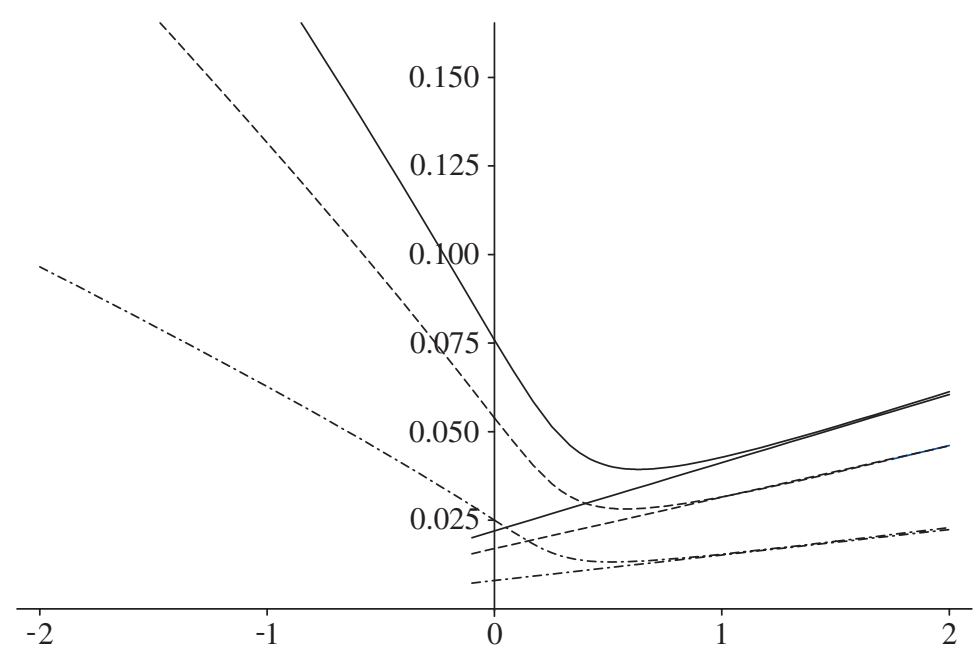

Figure 3: The Heston model. Parameters from [12]. The total implied variance and slopes for three maturities $t=0.4$ (dash-dot line), 0.9 (dashed line), and 1.3 (solid line) years.

When $\rho>0$, which is of little practical importance (at least in equity markets), the MGF may explode at a different point (see [2]), but Criterion 2 will still be satisfied. See Figure 3 for a numerical example.

\section{Acknowledgements}

The authors would like to thank L. C. G. Rogers, N. H. Bingham, and P. Embrechts for some related discussions.

\section{References}

[1] Albin, J. M. P. And Bengtsson, M. (2008). On the asymptotic behaviour of Lévy processes. Part I: Subexponential and exponential processes. To appear in Stoch. Process. Appl.

[2] Andersen, L. B. G. And Piterbarg, V. V. (2005). Moment explosions in stochastic volatility models. Preprint. Available at http://ssrn.com/abstract=559481.

[3] Barndorff-Nielsen, O., Kent, J. And Sørensen, M. (1982). Normal variance-mean mixtures and $z$ distributions. Internat. Statist. Rev. 50, 145-159.

[4] Benaim, S. And Friz, P. K. (2008). Regular variation and smile asymptotics. To appear in Math. Finance.

[5] Bingham, N. H., Goldie, C. M. And Teugels, J. L. (1987). Regular Variation. Cambridge University Press.

[6] Cont, R. and Tankov, P. (2004). Financial Modelling with Jump Processes. CRC Press, Boca Raton, FL.

[7] Gatheral, J. (2006). The Volatility Surface, A Practitioner's Guide. John Wiley, New York.

[8] LEE, R. (2004). The moment formula for implied volatility at extreme strikes. Math. Finance 14, 469-480.

[9] Nakagawa, K. (2006). Application of Tauberian theorem to the exponential decay of the tail probability of a random variable. Available at http//:arxiv.org/abs/math/0602569.

[10] Revuz, D. AND Yor, M. (1989). Continuous Martingales and Brownian Motion. Springer, Berlin.

[11] Schoutens, W. (2003). Lévy Processes in Finance. John Wiley, New York.

[12] Schoutens, W., Simons, E. And Tistaert, J. (2004). A perfect calibration! Now what? Wilmott Magazine, March 2004. 